\title{
Persistence of circulating endothelial microparticles in COPD despite smoking cessation
}

\author{
Yael Strulovici-Barel, ${ }^{1}$ Michelle R Staudt, ${ }^{1}$ Anja Krause, ${ }^{1}$ Cynthia Gordon, ${ }^{1}$ Ann E Tilley, \\ Ben-Gary Harvey, ${ }^{1,2}$ Robert J Kaner, ${ }^{1,2}$ Charleen Hollmann, ${ }^{1}$ Jason G Mezey, ${ }^{1,3}$ \\ Hans Bitter, ${ }^{4,5}$ Sreekumar G Pillai, ${ }^{4,6}$ Holly Hilton, ${ }^{4,7}$ Gerhard Wolff, ${ }^{4,8}$ \\ Christopher S Stevenson, ${ }^{4}$ Sudha Visvanathan, ${ }^{4,9}$ Jay S Fine, ${ }^{4,9}$ Ronald G Crystal $^{1}$
}

\begin{abstract}
- Additional material is published online only. To view please visit the journal online (http://dx.doi.org/10.1136/ thoraxjnl-2015-208274).
\end{abstract}

For numbered affiliations see end of article.

\section{Correspondence to} Dr Ronald G Crystal, Department of Genetic Medicine, Weill Cornell Medical College, 1300 York Avenue, Box 164, New York, NY 10065, USA; geneticmedicine@med.cornell. edu

Received 4 January 2016 Revised 21 April 2016 Accepted 27 April 2016 Published Online First 26 July 2016

\section{ABSTRACT}

Introduction Increasing evidence links COPD

pathogenesis with pulmonary capillary apoptosis. We previously demonstrated that plasma levels of circulating microparticles released from endothelial cells (EMPs) due to apoptosis are elevated in smokers with normal spirometry but low diffusion capacity, that is, with early evidence of lung destruction. We hypothesised that pulmonary capillary apoptosis persists with the development of COPD and assessed its reversibility in healthy smokers and COPD smokers following smoking cessation.

Methods Pulmonary function and high-resolution CT (HRCT) were assessed in 28 non-smokers, 61 healthy smokers and 49 COPD smokers; 17 healthy smokers and 18 COPD smokers quit smoking for 12 months following the baseline visit. Total EMP $\left(\mathrm{CD} 42 \mathrm{~b}^{-} \mathrm{CD} 31^{+}\right)$, pulmonary capillary EMP $\left(C D 42 b^{-} \mathrm{CD} 31^{+} A C E^{+}\right)$and apoptotic EMP $\left(\mathrm{CD} 42 \mathrm{~b}^{-} \mathrm{CD}_{2} \mathrm{E}^{+} / \mathrm{CD} 42 \mathrm{~b}^{-} \mathrm{CD} 31^{+}\right)$levels were quantified by flow cytometry.

Results Compared with non-smokers, healthy smokers and COPD smokers had elevated levels of circulating EMPs due to active pulmonary capillary endothelial apoptosis. Levels remained elevated over 12 months in healthy smokers and COPD smokers who continued smoking, but returned to non-smoker levels in healthy smokers who quit. In contrast, levels remained significantly abnormal in COPD smokers who quit.

Conclusions Pulmonary capillary apoptosis is reversible in healthy smokers who quit, but continues to play a role in COPD pathogenesis in smokers who progressed to airflow obstruction despite smoking cessation.

Trial registration number NCT00974064;

NCT01776398.

\section{INTRODUCTION}

COPD, the third leading cause of mortality in the USA, is defined by the Global Initiative for Chronic Obstructive Lung Disease (GOLD) as a chronic lung disorder with airflow limitation that is not fully reversible. ${ }^{1}$ There is overwhelming evidence that most cases of COPD are caused by cigarette smoking, with approximately $20 \%$ of smokers at risk for COPD if they continue to smoke. ${ }^{2}$ The airflow obstruction that characterises COPD is caused by a variable mixture of small airway disease (bronchitis) and parenchymal destruction (emphysema). ${ }^{13}$ Although the airway and alveolar diseases were classically considered as separate

\section{Key messages}

\section{What is the key question?}

- Is pulmonary capillary apoptosis, as measured by plasma levels of microparticles released from apoptotic endothelial cells (EMPs), reversible in healthy smokers and COPD smokers following smoking cessation?

\section{What is the bottom line?}

- Pulmonary capillary apoptosis is reversible in healthy smokers who quit, but not in COPD smokers despite 12 months of smoking cessation, suggesting that the apoptosis continues to play a role in COPD pathogenesis in smokers who progressed to airflow obstruction despite smoking cessation.

\section{Why read on?}

- Our longitudinal study demonstrates persistent endothelial stress in subjects with COPD despite smoking cessation and provides a biological correlate to the epidemiological data showing that smoking cessation only has a moderate effect on the continuous decline of lung function in COPD smokers, suggesting EMP levels might serve as a useful biomarker to follow smoking-associated endothelial apoptosis.
- http://dx.doi.org/10.1136/ thoraxjnl-2016-208993

CrossMark

\footnotetext{
To cite: Strulovici-Barel $Y$, Staudt MR, Krause A, et al. Thorax 2016;71:1137-
} 1144. entities, it is now recognised that they usually coexist to variable degrees and are closely linked, with the parenchymal destruction evolving around areas of small airway disease. ${ }^{3-7}$

There is increasing evidence that the pathogenesis of COPD is linked, in part, to apoptosis of pulmonary capillaries. ${ }^{8-11}$ Consistent with this concept, we recently demonstrated that smokers and, to a greater extent, smokers with early evidence of lung destruction (normal spirometry, but low diffusing capacity (DLCO)) have elevated levels of circulating endothelial microparticles (EMPs). ${ }^{12}$ Importantly, a significant proportion of these EMPs are derived from pulmonary capillaries and have characteristics of apoptotic EMPs, that is, they are derived from lung endothelial cells that have been induced to undergo apoptosis. ${ }^{12-14}$ 
If elevated levels of circulating apoptotic EMPs are a reflection of active smoking-related injury, to lung endothelium, based on the knowledge that even those COPD smokers who stop smoking continue to have a decline in lung function that is more rapid than that of healthy non-smokers or healthy smokers who quit smoking, ${ }^{15}$ we hypothesised that elevated levels of circulating apoptotic EMPs may persist in COPD smokers following smoking cessation, reflecting continuous lung endothelial injury that persists even after the stress of smoking is removed. To assess this hypothesis, we quantified the levels of circulating EMPs, and the fraction represented by apoptotic EMPs, in non-smokers, healthy smokers and smokers with COPD at baseline and at three more intervals over 1 year and then compared those levels with those obtained from a subgroup of healthy smokers and COPD smokers who successfully stopped smoking after baseline assessment. The data demonstrate that circulating EMP levels derived from apoptotic pulmonary capillary endothelial cells remain elevated over 1 year in healthy smokers and COPD smokers who continue smoking. However, while levels of total and apoptotic EMPs return to non-smoker levels in healthy smokers who successfully quit smoking, total and apoptotic EMP levels remain elevated in COPD smokers who quit smoking persisting 12 months of smoking cessation.

\section{METHODS}

\section{Human subjects and clinical phenotypes}

All subjects were evaluated at the Weill Cornell NIH Clinical and Translational Science Center and Department of Genetic Medicine Clinical Research Facility, under the clinical protocols approved by the Institutional Review Board. Recruitment was from the general population in New York City by posting advertisements in local newspapers and on electronic bulletin boards. All subjects provided written consent prior to enrolment and then underwent thorough medical history, screening and pulmonary function tests. Smoking status was determined based on self-reported history and quantified levels of urine nicotine metabolites. For details and full inclusion/exclusion criteria, see online supplementary methods. A total of 138 subjects were assessed for circulating total and apoptotic EMP levels at baseline, 3, 6 and 12 months (28 non-smokers, 61 healthy smokers and 49 COPD GOLD I/II smokers). See online supplementary figure $\mathrm{S} 1$ for study design.

\section{Characterisation of plasma EMPs}

EMPs were quantified according to a standard operating procedure as previously described ${ }^{12}$ to eliminate variability in sample processing. Briefly, blood was collected, processed within 1 hour and stained for the endothelial markers PECAM (CD31) and E-selectin (CD62E) and the constitutive platelet-specific glycoprotein $\mathrm{Ib}(\mathrm{CD} 42 \mathrm{~b})$ to differentiate endothelium-originated microparticles from platelet-derived microparticles, which also express CD31. EMPs were defined as microparticles $<1.5 \mu \mathrm{m}$ in size, expressing $\mathrm{CD}_{3} 1^{+}$or $\mathrm{CD} 62 \mathrm{E}^{+}$but not $\mathrm{CD} 42 \mathrm{~b}$. We have previously shown that staining with annexin $\mathrm{V}$ is comparable with $\mathrm{CD} 42 \mathrm{~b}^{-} \mathrm{CD} 31^{+}$staining, ${ }^{12}$ but annexin $\mathrm{V}$ was not used because it is not specific for EMPs. ${ }^{16}$ Circulating EMPs are present in low levels in plasma of healthy subjects, reflecting normal endothelial turnover, ${ }^{17}$ but their levels increase in a variety of vascular-related disorders. As in our previous study, ${ }^{12}$ total EMP levels above the non-smoker total EMP mean level plus 2 SDs were considered abnormally elevated. To assess the presence of relative contribution of pulmonary capillary endothelium to the elevated total EMP levels, ${ }^{1}{ }^{12}$ EMPs were co-stained with antihuman ACE inhibitors, which is abundantly expressed on pulmonary capillary endothelium $\left(\mathrm{CD} 42 \mathrm{~b}^{-} \mathrm{CD} 31^{+} \mathrm{ACE}^{+}\right){ }^{13}$ To quantify the proportion of EMPs that originated from apoptotic endothelium, we assessed the ratio of $\mathrm{CD}_{2} \mathrm{~b}^{-} \mathrm{CD} 62 \mathrm{E}^{+} / \mathrm{CD} 42 \mathrm{~b}^{-} \mathrm{CD} 31^{+}$EMPs in all groups. EMPs induced by apoptosis express the constitutive CD31 marker, whereas activation-induced EMPs express CD62E. Using these criteria, EMPs with a low $\mathrm{CD} 42 \mathrm{~b}^{-} \mathrm{CD} 62 \mathrm{E}^{+}$to $\mathrm{CD} 42 \mathrm{~b}^{-} \mathrm{CD} 31^{+}$ratio were defined as 'apoptotic EMPs', and the percentage of subjects with apoptotic EMPs with $\mathrm{CD}_{2} 2 \mathrm{~b}^{-} \mathrm{CD} 62 \mathrm{E}^{+} / \mathrm{CD} 42 \mathrm{~b}^{-} \mathrm{CD} 31^{+}$ratio below the lowest ratio in healthy non-smokers was quantified. See online supplementary methods for further details on the EMP analysis.

\section{Assessment after smoking cessation}

After the baseline levels of total and apoptotic EMPs were determined, all healthy smokers and COPD smokers were invited to stop smoking using a combination of varenicline and counselling for 3 months (see details in online supplementary methods). A total of 17 healthy smokers and 18 COPD smokers successfully quit smoking as confirmed by urine tobacco metabolite level quantification at 3,6 and 12 months after the baseline; subjects were considered true quitters only if there were no detectable levels of nicotine metabolites in the urine at months 3, 6 and 12. Healthy smokers and smokers with COPD were treated with exact same prescription of varenicline to prevent any effect it might have on EMP levels. All other healthy smokers and COPD smokers were considered current smokers if urine cotinine level was $\geq 104 \mathrm{ng} / \mathrm{mL}$ at each time point, a level based on our previous study of low-level smoke exposure, ${ }^{18}$ where $104 \mathrm{ng} / \mathrm{mL}$ was calculated as the induction half-maximal level $\left(\mathrm{ID}_{50}\right)$ at which the small airway epithelium, the initial site of smoking-related pathology, showed an abnormal response. See online supplementary figure S1 for study design.

\section{Statistical analysis}

$\chi^{2}$ test, with a Yates' correction for small sample size, was used for comparing demographic parameters and the number of subjects with high total EMP and apoptotic EMP levels, and pairwise analysis of variance was used to compare total and apoptotic EMP levels between groups and within a group, at different time points with no correction for multiple test, as the number of tests was low $(<21)$. In order to eliminate the effect of diseases known to be associated with elevated EMPs, including diabetes ${ }^{19} 20$ and systemic hypertension, ${ }^{21}$ or drugs for COPD, including corticosteroids and bronchodilators, subjects with known disease state or drug treatment were removed from statistical analysis. Removal of those subjects did not alter the results.

\section{RESULTS}

\section{Study population}

Except for minor differences, the study population of nonsmokers was comparable with the healthy smokers and COPD smokers in all demographic parameters (see table 1 for details). At each time point, the non-smokers had undetectable urine nicotine (not shown) and cotinine levels (figure 1). Both the healthy smokers and COPD smokers who continued smoking had urine cotinine levels consistent with tobacco smoking and comparable in both groups at each time point ( $p>0.07$, all comparisons). In healthy smokers and COPD smokers who quit smoking, urine nicotine and cotinine levels were consistent with smoking at baseline and were undetectable at all time points 
Table 1 Study Population*

\begin{tabular}{|c|c|c|c|c|c|c|c|}
\hline \multirow[b]{2}{*}{ Parameter } & \multirow[b]{2}{*}{ Non-smokers } & \multicolumn{3}{|c|}{ Healthy smokerst } & \multicolumn{3}{|c|}{ COPD smokers $\ddagger$} \\
\hline & & All & $\begin{array}{l}\text { Who continued } \\
\text { smoking }\end{array}$ & Who quit & All & $\begin{array}{l}\text { Who continued } \\
\text { smoking }\end{array}$ & Who quit \\
\hline $\mathrm{n}$ & 28 & 61 & 44 & 17 & 49 & 31 & 18 \\
\hline Gender (M/F) & $15 / 13$ & $47 / 14$ & $37 / 7$ & $10 / 7$ & $46 / 3$ & $30 / 1$ & $16 / 2$ \\
\hline Ethnicity $(\mathrm{B} / \mathrm{W} / 0) \S$ & 10/7/11 & $33 / 9 / 19$ & 23/7/14 & $10 / 2 / 5$ & $27 / 12 / 10$ & $18 / 6 / 7$ & $9 / 6 / 3$ \\
\hline Age & $37 \pm 11$ & $44 \pm 9$ & $44 \pm 9$ & $45 \pm 10$ & $53 \pm 8$ & $53 \pm 7$ & $53 \pm 9$ \\
\hline BMI & $27 \pm 5$ & $28 \pm 5$ & $27 \pm 4$ & $30 \pm 4$ & $27 \pm 4$ & $25 \pm 3$ & $29 \pm 5$ \\
\hline \multicolumn{8}{|l|}{ Smoking history } \\
\hline Pack-year & - & $23 \pm 12$ & $24 \pm 12$ & $20 \pm 8$ & $32 \pm 14$ & $32 \pm 15$ & $34 \pm 12$ \\
\hline Pack per day & - & $0.8 \pm 0.6$ & $1.0 \pm 0.6$ & $0.6 \pm 0.2$ & $0.8 \pm 0.4$ & $0.8 \pm 0.5$ & $0.8 \pm 0.3$ \\
\hline Age of initiation & - & $16 \pm 3$ & $16 \pm 3$ & $16 \pm 3$ & $16 \pm 3$ & $16 \pm 3$ & $16 \pm 3$ \\
\hline Urine cotinine (ng/mL) & - & $1693 \pm 961$ & $1828 \pm 930$ & $1323 \pm 979$ & $1747 \pm 980$ & $1953 \pm 959$ & $1393 \pm 938$ \\
\hline Subjects with emphysema (n, \%)ף & $1(4 \%)$ & $0(0 \%)$ & $0(0 \%)$ & $0(0 \%)$ & $13(27 \%)$ & $9(29 \%)$ & $4(22 \%)$ \\
\hline \multicolumn{8}{|l|}{ Pulmonary function** } \\
\hline FEV1 & $106 \pm 11$ & $109 \pm 11$ & $109 \pm 10$ & $107 \pm 13$ & $85 \pm 16$ & $87 \pm 16$ & $82 \pm 17$ \\
\hline FVC & $107 \pm 11$ & $111 \pm 10$ & $110 \pm 10$ & $111 \pm 11$ & $108 \pm 16$ & $109 \pm 17$ & $105 \pm 15$ \\
\hline FEV1/FVC & $83 \pm 5$ & $80 \pm 5$ & $81 \pm 4$ & $79 \pm 6$ & $63 \pm 6$ & $64 \pm 6$ & $63 \pm 7$ \\
\hline $\mathrm{TLC}$ & $99 \pm 16$ & $96 \pm 12$ & $95 \pm 12$ & $96 \pm 10$ & $99 \pm 12$ & $100 \pm 13$ & $99 \pm 10$ \\
\hline DLCO & $91 \pm 11$ & $89 \pm 8$ & $89 \pm 9$ & $90 \pm 6$ & $71 \pm 14$ & $68 \pm 13$ & $77 \pm 15$ \\
\hline GOLD stage (I/II) & - & - & - & - & $31 / 18$ & $20 / 11$ & $11 / 7$ \\
\hline \multicolumn{8}{|c|}{ 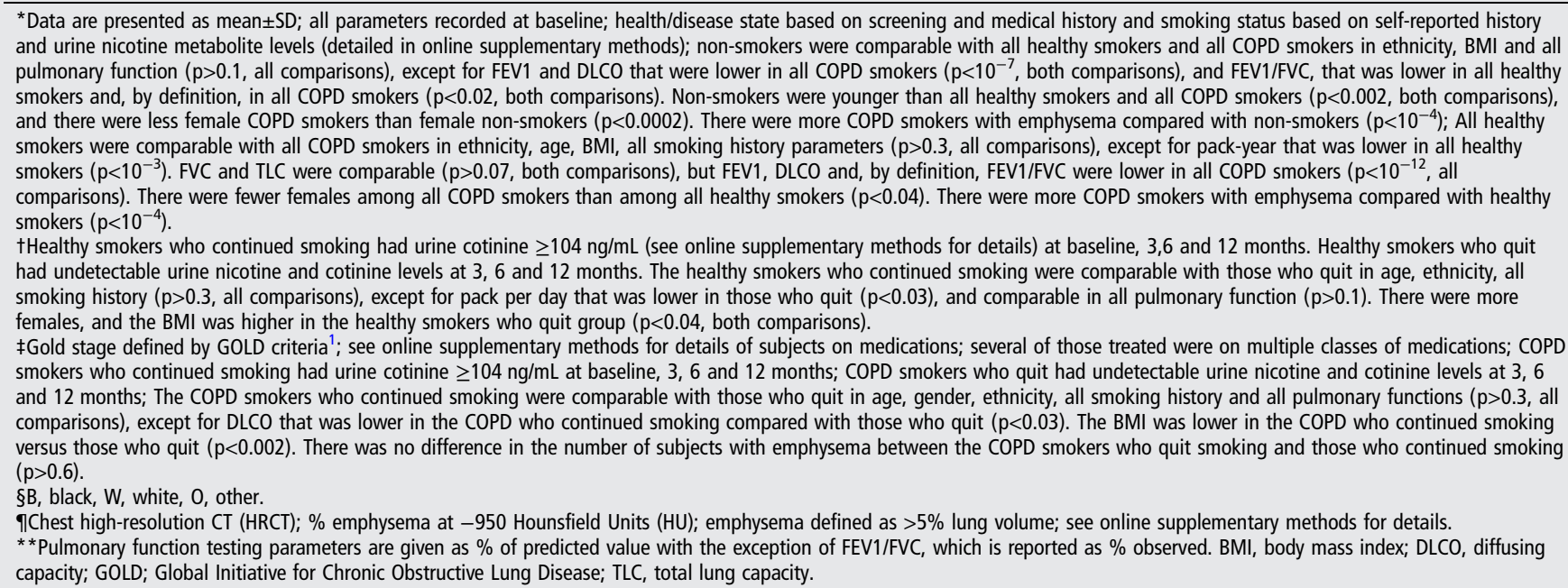 } \\
\hline
\end{tabular}

after baseline (see online supplementary methods for details of urine nicotine metabolite level criteria for smoking/abstinence).

\section{Total EMP levels}

Consistent with our prior study with a different cohort, ${ }^{12}$ total EMP levels were higher in healthy smokers compared with nonsmokers (figure $2 \mathrm{~A}, \mathrm{p}<0.005$ ). In addition, COPD smokers had elevated levels of total EMPs compared with non-smokers $(\mathrm{p}<0.007)$, but lower than those of healthy smokers $(\mathrm{p}<0.02)$. Twenty-two (36\%) healthy smokers and eight (16\%) COPD smokers had high levels of total EMPs $(p<0.03)$. There was no correlation between the level of total EMPs and any pulmonary function or demographic parameters $\left(r^{2}<0.08\right.$, all correlations, see online supplementary figure S2). For the COPD group, total circulating EMP levels were independent of drugs used for treatment, including inhaled and systemic corticosteroids and bronchodilators.

\section{Origin of the circulating EMPs}

In our prior study of circulating EMPs, ${ }^{12}$ we demonstrated that most of the circulating CD $42 \mathrm{~b}^{-} \mathrm{CD} 31^{+}$EMPs were positive for
ACE inhibitors, a surface protein more highly expressed on pulmonary capillary endothelium than in other endothelial beds. ${ }^{13}$ In the present study, an average of $75 \%$ of the circulating EMPs in all subjects were $\mathrm{CD} 42 \mathrm{~b}^{-} \mathrm{CD} 31^{+} \mathrm{ACE}^{+}$. There were similar levels of $\mathrm{ACE}^{+}$EMPs in the healthy smoker group compared with the non-smokers or COPD smokers (figure 2B, $\mathrm{p}>0.1$, both comparisons), and higher levels of $\mathrm{ACE}^{+} \mathrm{EMPs}$ in the COPD smoker group compared with the non-smokers $(\mathrm{p}<0.0001)$.

\section{EMPs derived from apoptotic endothelium}

To quantify the proportion of EMPs originating from apoptotic endothelium, we assessed the ratio of $\mathrm{CD}_{2} 2 \mathrm{~b}^{-} \mathrm{CD} 62 \mathrm{E}^{+} /$ $\mathrm{CD} 42 \mathrm{~b}^{-} \mathrm{CD} 31^{+}$EMPs in all groups. The $\mathrm{CD}_{2} 2 \mathrm{~b}^{-} \mathrm{CD} 62 \mathrm{E}^{+} /$ $\mathrm{CD} 42 \mathrm{~b}^{-} \mathrm{CD} 31^{+}$EMP ratio in non-smokers was distributed around a mean of 0.9 , significantly higher than that in healthy smokers (mean 0.6; a lower ratio indicates greater number of apoptotic EMPs) and COPD smokers (mean 0.55, p <0.0001, both groups compared with non-smokers; $p>0.6$, COPD smokers compared with healthy smokers). $\mathrm{CD}_{2} 2 \mathrm{~b}^{-} \mathrm{CD} 62 \mathrm{E}^{+} /$ 


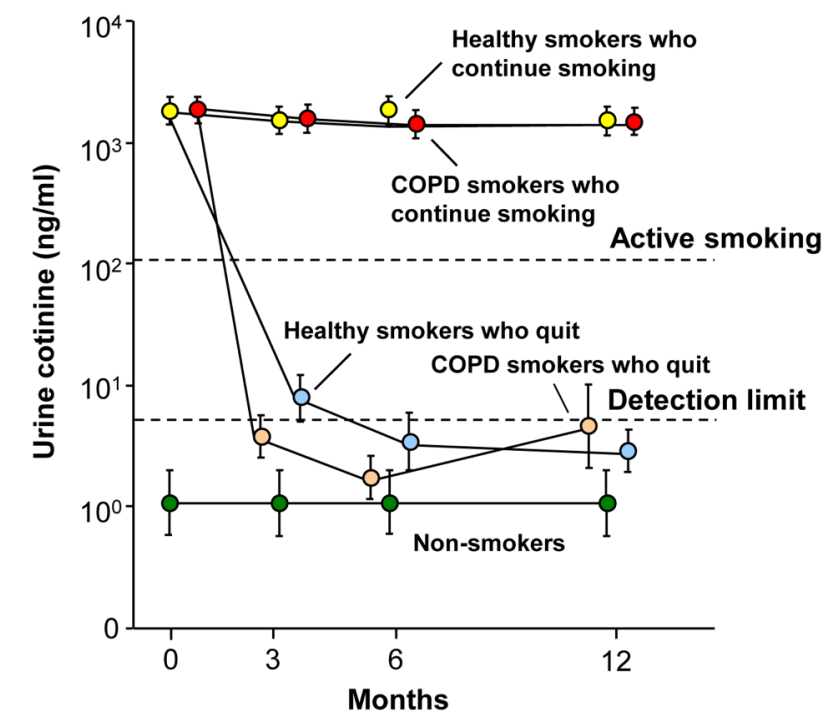

Figure 1 Urine cotinine levels $(\mathrm{ng} / \mathrm{mL})$ as a measure of smoking status at baseline and at 3, 6 and 12 months in non-smokers, healthy smokers and smokers with COPD (COPD smokers). Shown are data for non-smokers ( $n=28$, green circles), healthy smokers who continue to smoke ( $n=44$, yellow circles), healthy smokers who quit smoking following baseline ( $n=17$, light blue circles), COPD smokers who continue to smoke ( $n=31$, red circles) and COPD smokers who quit smoking following baseline $(n=18$, tan circles). Data represent mean \pm SE. Dashed lines indicate urine cotinine detection level of $\leq 5 \mathrm{ng} / \mathrm{mL}$ and urine cotinine level of $\geq 104 \mathrm{ng} / \mathrm{mL}$ for active smoking (see online supplementary methods). EMP, endothelial microparticles.

$\mathrm{CD} 42 \mathrm{~b}^{-} \mathrm{CD} 31^{+}$EMPs below the lowest level in non-smokers were defined as apoptotic EMPs with $48 \%$ of healthy smokers and $45 \%$ of COPD smokers having increased levels of apoptotic EMPs $(p>0.7)$, that is, even though there are less subjects with total circulating EMPs in COPD smokers compared with healthy smokers (figure 2A), the relative proportion of subjects with apoptotic EMPs was similar (figure 2C), implying that there is active pulmonary capillary apoptosis ongoing in both the healthy smokers and COPD smokers. There was no correlation of $\mathrm{CD} 42 \mathrm{~b}^{-} \mathrm{CD} 62 \mathrm{E}^{+} / \mathrm{CD} 42 \mathrm{~b}^{-} \mathrm{CD} 31^{+}$EMP ratio with any lung function or demographic parameter $\left(r^{2}=0.09\right.$, all correlations, see online supplementary figure S3). Within the COPD smoker group, there was no correlation of total CD42b $-\mathrm{CD} 31^{+}$EMP levels or $\mathrm{CD} 42 \mathrm{~b}^{-} \mathrm{CD} 62 \mathrm{E}^{+} / \mathrm{CD} 42 \mathrm{~b}^{-} \mathrm{CD} 31^{+}$ EMP ratio to the DLCO (\% predicted) or $\%$ emphysema on high-resolution CT (HRCT) $\left(r^{2}=0.04\right.$, all comparisons; not shown), suggesting these parameters are likely measuring different aspects of the destruction process.

\section{Effect of smoking cessation on total EMP levels}

The levels of total EMPs were followed for a period of 1 year at baseline, 3, 6 and 12 months in non-smokers, healthy smokers and COPD smokers (figure 3A, B). In non-smokers, total EMP levels were stably low throughout the duration of the study ( $p>0.6$, each time point compared with baseline). In healthy smokers who continued smoking, the levels were stably high at each time point $(p>0.1$, each time point compared with baseline; $p<0.05$, each time point compared with the non-smokers at the same time point). In contrast, in the healthy smokers who quit smoking, total EMP levels significantly decreased following smoking cessation to the levels of non-smokers $(p<0.002$, each time point compared with baseline; $p>0.4$, compared with non-smokers at the same time point at 3, 6 and 12 months, figure 3A). At 12 months, the total EMP levels in healthy smokers who continued smoking were significantly higher compared with healthy smokers who quit $\left(p<10^{-3}\right)$. Total EMP levels in COPD smokers who continued smoking were stably elevated compared with non-smokers at each time point ( $p<0.05$, all comparisons). In contrast to the healthy smokers, in COPD smokers who quit smoking, total EMP levels initially decreased following smoking cessation at month 3 , but became elevated again at 6 and 12 months (figure 3B). The levels were not significantly different compared with non-smokers at 3 and 6 months ( $p>0.1$, both comparisons), but were significantly elevated at 12 months $(\mathrm{p}<0.05)$ and similar to those of COPD smokers who continued smoking $(\mathrm{p}>0.08)$.

\section{Effect of smoking cessation on apoptotic EMP levels}

The ratio of $\mathrm{CD} 42 \mathrm{~b}^{-} \mathrm{CD} 62 \mathrm{E}^{+} / \mathrm{CD} 42 \mathrm{~b}^{-} \mathrm{CD} 31^{+}$EMPs was stably high in non-smokers and stably low (ie, EMPs were apoptoticderived) in healthy smokers who continued smoking ( $p>0.1$, each time point compared with baseline within the non-smoker group and within the healthy smoker group; $\mathrm{p}<0.01$, healthy smokers who continued smoking compared with non-smokers, at baseline, months 6 and 12; figure 3C). Interestingly, in the healthy smokers who quit smoking group, the ratio increased following smoking cessation $\left(\mathrm{p}<10^{-3}\right.$, within the healthy smokers who quit group, at months 3 and 6 compared with baseline, a $p$ value significant even with correction for multiple tests) to the level of non-smokers at months 3 and 6 ( $p>0.1$, both comparisons compared with non-smokers at the same time point) and superseded that of non-smokers at month $12(\mathrm{p}<0.05)$. The ratio was significantly higher (ie, less apoptotic-derived EMPs) in healthy smokers who quit smoking compared with smokers who continued smoking at month $12\left(\mathrm{p}<10^{-3}\right.$, a $\mathrm{p}$ value significant even with correction for multiple tests). There were no significant changes in the $\mathrm{CD}_{2} \mathrm{~b}^{-} \mathrm{CD} 62 \mathrm{E}^{+} / \mathrm{CD} 42 \mathrm{~b}^{-} \mathrm{CD} 31^{+}$ratio in COPD smokers who continued smoking $(p>0.1$, within the COPD smoker group, each time point compared with baseline), and it remained significantly low compared with non-smokers at each time point $(\mathrm{p}<0.01$, all comparisons, figure $3 \mathrm{D})$. In contrast to the healthy smoker group, in COPD smokers who quit smoking, there was no change in the ratio $(p>0.1$, within the COPD who quit group, each time point compared with baseline), and the ratio remained significantly low compared with non-smokers at baseline and month $12(\mathrm{p}<0.05$, both comparisons). The ratio at month 12 was similarly low (ie, more apoptotic-derived EMPs) in COPD smokers who continued smoking and in COPD smokers who quit $(p>0.4)$.

\section{DISCUSSION}

COPD is a chronic, debilitating disease that is caused primarily by cigarette smoking. ${ }^{13-5}$ Cigarette smoke is very complex, with $10^{14}$ oxidants and $>4000$ compounds stressing the lung with each puff. ${ }^{22}$ The apoptotic loss of pulmonary capillaries in association with smoking is well recognised, ${ }^{8-10}$ although it is not known whether this represents the primary mechanism of lung destruction associated with smoking, a subtype of lung destruction, or is secondary to other mechanisms, such as inflammatory cell-mediated processes. ${ }^{5} 691023$ Using circulating, total and apoptotic-endothelial cell microparticles as biomarkers for pulmonary capillary apoptosis, the data in the present study document that pulmonary capillary endothelial apoptosis is a persistent process in smokers with and without COPD. In healthy smokers who quit smoking, the levels of total and apoptotic EMPs return to the levels of non-smokers over time. In contrast, in COPD smokers who quit smoking, the levels of total and 
Figure 2 Levels of total circulating endothelium microparticles (EMPs), $\mathrm{ACE}^{+}$EMPs and apoptotic EMPs in plasma at baseline. Shown are data for non-smokers ( $n=28$, green circles), healthy smokers ( $n=61$, yellow circles) and smokers with COPD ( $n=49$, red circles). (A) Total CD42 $\mathrm{b}^{-} \mathrm{CD} 31^{+}$EMPs. The grey shaded area indicates the non-smoker mean \pm 2 SDs. The $\%$ value above the smoker populations represents the proportion of smokers with EMP levels above that mean. (B) $\mathrm{CD}_{22 \mathrm{~b}^{-} \mathrm{CD} 31^{+} \mathrm{ACE}}^{+}$EMPs. Proportion of total CD42 $b^{-} \mathrm{CD} 31^{+}$ EMPs in plasma that express $\mathrm{ACE}^{+}$. The grey shaded area represents the non-smoker mean \pm 2 SDs. (C) Ratio of EMPs. The dashed line indicates the lowest ratio of $\mathrm{CD}_{22} \mathrm{~b}^{-} \mathrm{CD} 2 \mathrm{E}^{+}$/ $\mathrm{CD} 42 \mathrm{~b}^{-} \mathrm{CD} 31^{+}$EMPs in non-smokers. The $\%$ value below the smoker populations represents the proportion of smokers with a ratio below that level. $(A-C)$ Bold dashed lines represent the mean for each group. Symbols inside the dots: A horizontal line indicates subjects with systemic hypertension; a vertical line indicates subjects with type 2 diabetes mellitus; a star indicates subjects with type 1 diabetes.

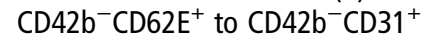

A

Total EMPs at baseline

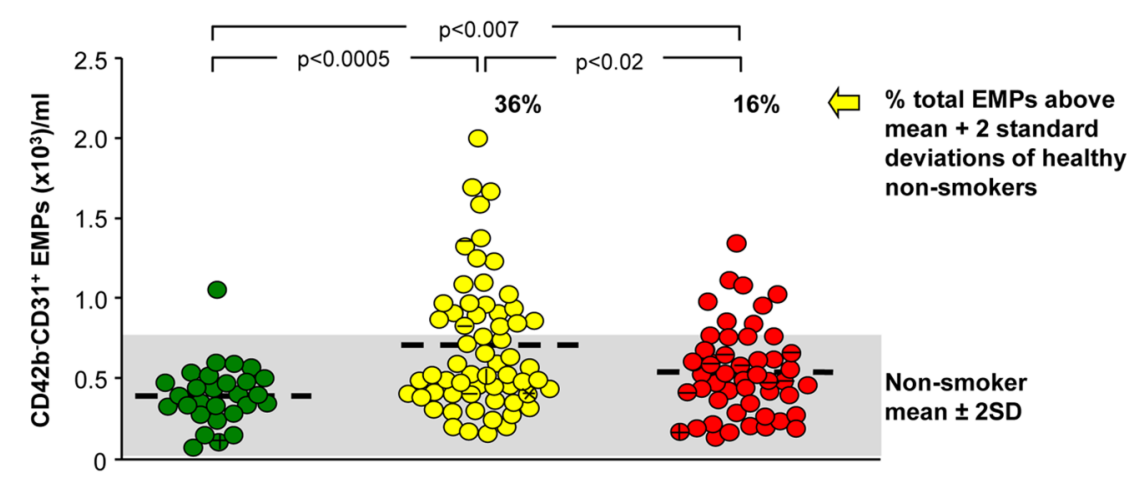

B $\quad \% \mathrm{ACE}^{+}$Total EMPs at baseline

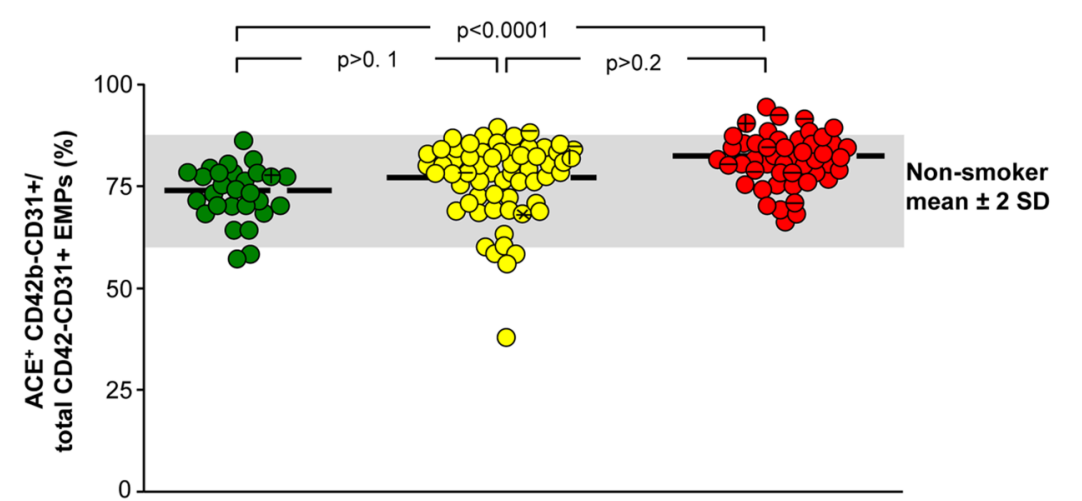

C

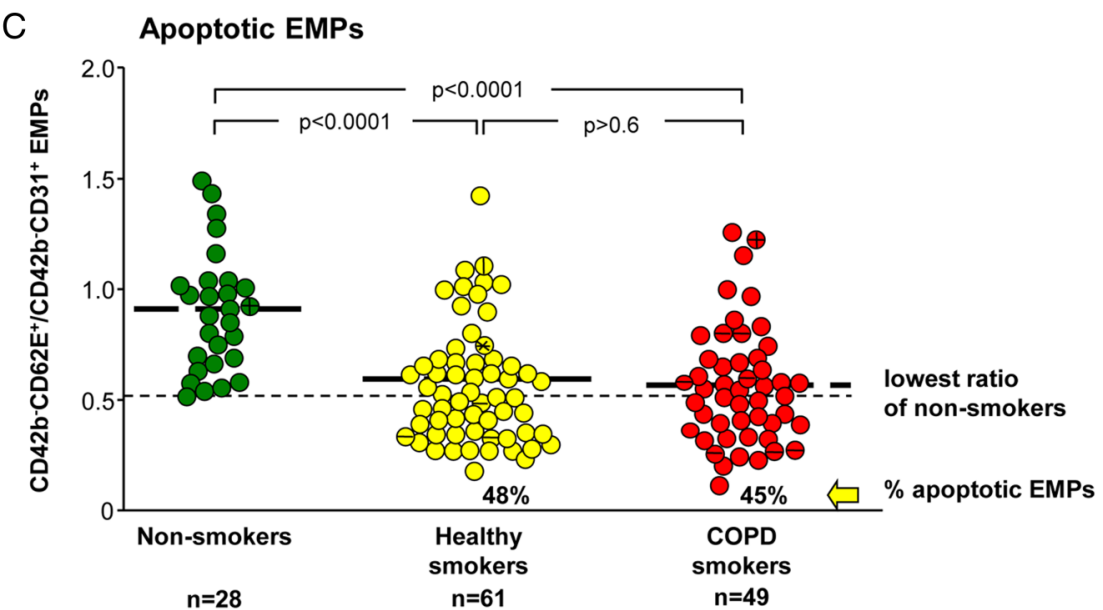

apoptotic EMPs remain abnormal over 1 year and were still significantly different compared with non-smoker levels at 12 months. The majority of the COPD subjects assessed for EMPs in this study were GOLD I and GOLD II, providing evidence for ongoing pulmonary endothelial apoptosis even in the earliest stages of COPD. Importantly, the FEV1/FVC ratio of the COPD smokers was $0.63 \pm 0.06$, on average, well below the 0.7 ratio threshold definition of COPD GOLD I.

These observations were not altered by the removal of subjects with diseases known to be associated with elevated EMPs, suggesting that smoking has a much stronger effect on EMP levels than hypertension or diabetes.

\section{Endothelial microparticles}

Different cell types respond to cell activation, injury and/or apoptosis by shedding submicron membrane vesicles, called microparticles, from their plasma membranes. ${ }^{16} 24$
Microparticles detected in plasma are of various cellular origins, predominantly derived from platelets, leucocytes and endothelial cells. ${ }^{24}$ Endothelial microparticles, defined as $\mathrm{CD}_{2} \mathrm{~b}^{-} \mathrm{CD} 31^{+}$or $\mathrm{CD} 42 \mathrm{~b}^{-} \mathrm{CD} 62 \mathrm{E}^{+}$microparticles, can be generally distinguished from microparticles of other cell types by their size $(0.1-1.5 \mu \mathrm{m})$, constitutive expression of the platelet-endothelial cell adhesion marker CD31 (PECAM) and the absence of the platelet-specific glycoprotein Ib marker CD42b. ${ }^{24}{ }^{25}$ Apoptosis-induced EMPs express CD31, whereas activation-induced EMPs express CD62E. In this regard, a low ratio of $\mathrm{CD} 42 \mathrm{~b}^{-} \mathrm{CD} 62 \mathrm{E}^{+}$to $\mathrm{CD} 42 \mathrm{~b}^{-} \mathrm{CD} 31^{+}$EMPs can be used as an index of apoptosis. ${ }^{24}{ }^{25}$ EMPs variably co-express phosphatidylserine (annexin V). ${ }^{25} 26$

EMPs can be found in the plasma of healthy subjects; ${ }^{24}$ however, increased levels are associated with vascular disease and endothelial dysfunction in atherosclerosis and acute coronary syndrome, ${ }^{24} 27$ acute ischaemic stroke, ${ }^{24} 28$ end-stage renal 
A
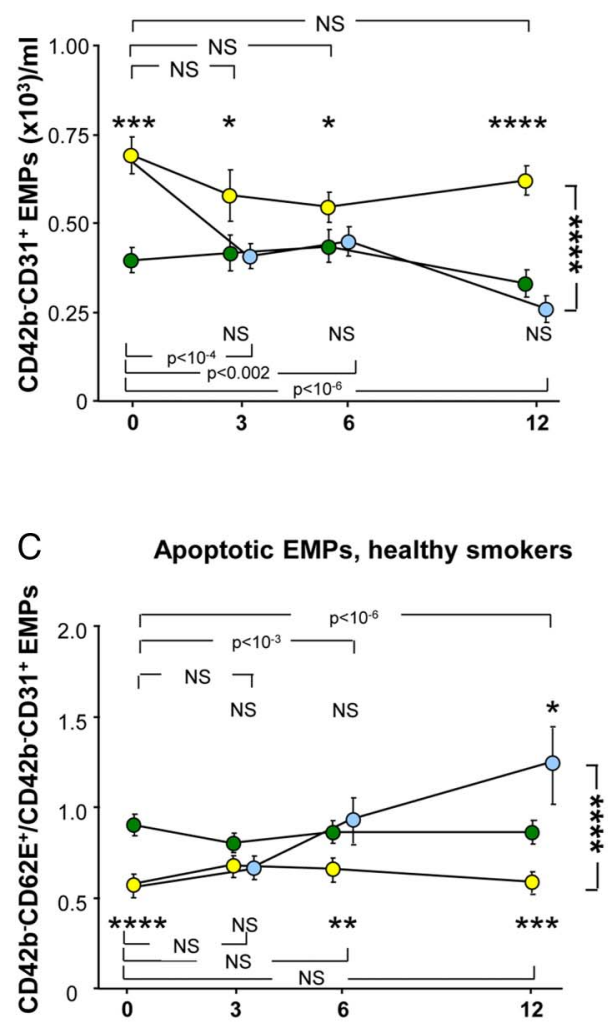

B Total EMPs, COPD smokers

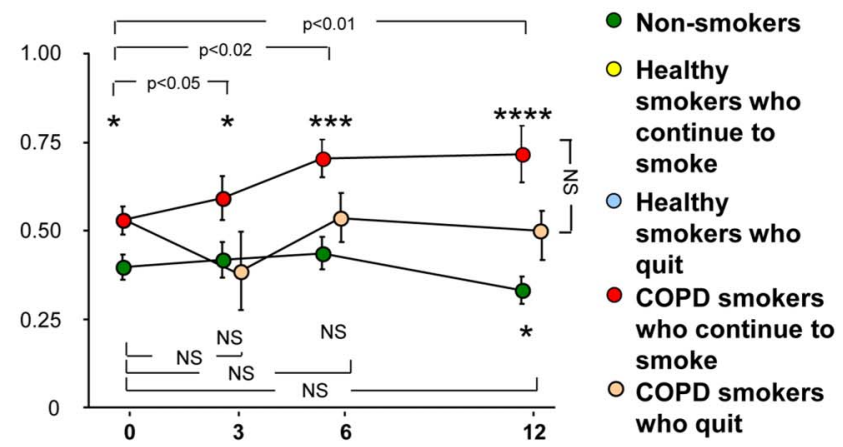

D Apoptotic EMPs, COPD smokers

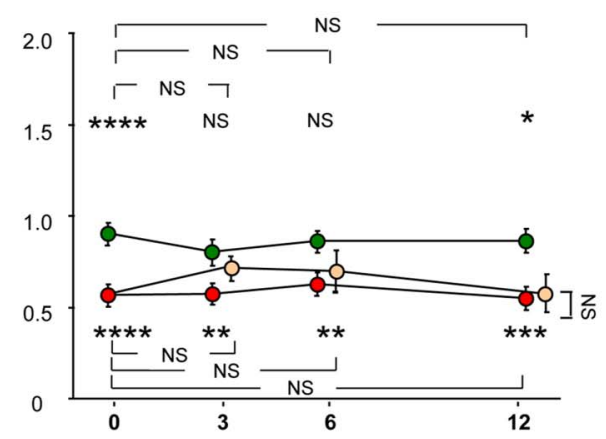

Figure 3 Total circulating $C D 42 \mathrm{~b}^{-} \mathrm{CD} 31^{+}$endothelial microparticles (EMPs) and ratio of $\mathrm{CD} 42 \mathrm{~b}^{-} \mathrm{CD} 62 \mathrm{E}^{+}$to $\mathrm{CD} 42 \mathrm{~b}^{-} \mathrm{CD} 31^{+}$EMPs over time in non-smokers ( $n=28$, green circles), healthy smokers who continue to smoke ( $n=44$, yellow circles), healthy smokers who quit smoking following baseline ( $\mathrm{n}=17$, light blue circles), smokers with COPD (COPD smokers) who continue smoking ( $\mathrm{n}=31$, red circles) and COPD smokers who quit smoking following baseline ( $n=18$, tan circles). ( $A$ and $B$ ) Total CD42b-CD31 EMPs. (C and D) Ratio of $C D 42 b^{-} C D 62 E^{+}$to $C D 42 b^{-} C D 31^{+}$EMPs. ( $A$ and $C)$. Healthy smokers who continue to smoke and healthy smokers who quit smoking versus non-smokers. (B and D) COPD smokers who continue to smoke and COPD smokers who quit smoking versus non-smokers. (A-D) Data represent mean \pm SE. p Values comparing each time point to baseline within the same group are shown at the top of the panel (for the group, ie, above the non-smokers at month 12) and at the bottom of the panel (for the group, ie, below the non-smokers at month 12). $p$ Values comparing each time point in a smoker group to the same time point in the non-smoker group are shown above the group, if the group is above the non-smokers at month 12 and below the group if the group is below the non-smokers at month 12. $p$ Values comparing the subjects who continue to smoke with those who quit smoking at month 12 are to the right of the panel. NS, not significant; ${ }^{*}{ }^{* *},{ }^{* * *},{ }^{* * * *}$ indicate $p<0.05, p<0.01, p<0.001$ and $p<0.0001$, respectively.

failure, ${ }^{29}$ pre-eclampsia and gestational hypertension, ${ }^{30}$ hypertension, ${ }^{21} 2429$ pulmonary hypertension, ${ }^{31}$ metabolic syndrome $^{32}$ venous thromboembolism ${ }^{33}$ and obstructive sleep apnoea. ${ }^{34}$ Consistent with our prior study ${ }^{12}$ and the data in the present study, Heiss and colleagues ${ }^{35}$ have shown healthy nonsmokers exposed for $30 \mathrm{~min}$ to low levels of cigarette smoke had increased circulating EMP levels.

\section{Clinical measures of alveolar capillary destruction}

The observation of endothelial apoptosis in the lungs of humans with emphysema is well documented. There is increased DNA fragmentation in the pulmonary capillaries and arteriolar endothelium of subjects with COPD, and increased alveolar endothelial and epithelial cell death in human emphysematous lungs compared with lungs of non-smokers or smokers without emphysema. ${ }^{9} 10$ Lung levels of alveolar epithelial-derived vascular endothelial growth factor are decreased in emphysema, contributing to the complex mechanisms of pulmonary capillary endothelial destruction. ${ }^{10}$

The data in the present study add total and apoptotic EMP levels to a growing list of biomarkers that may be useful in assessing active destruction and defining subclinical molecular phenotypes of lung disease. ${ }^{17} 36{ }^{37}$ As described in the editorial by Chandra and colleagues, ${ }^{14}$ accompanying our study of apoptotic EMPs in healthy smokers and smokers with normal spirometry but low DLCO, the observation of elevated levels of apoptotic EMPs may represent early lung destruction and a subphenotype of subjects with lung destruction. The observation in the present study that, on average, a fraction of smokers with COPD also have elevated levels of total and apoptotic EMPs is consistent with the discussion of the concept of vascular subphenotypes of COPD by Chandra et al. ${ }^{14}$ In this context, the data on circulating and apoptotic EMPs support the idea that, while the global concept of COPD as an FEV1-defined disorder is useful for epidemiologic studies and as a paradigm for routine clinical care, it is likely masking the concept that there are several subphenotypes of COPD. ${ }^{38-42}$ Consistent with this concept, correlation of the total EMP levels and apoptotic EMP levels with the conventional measures of lung destruction (DLCO and HRCT) was, at best, very weak. This may imply that, while overlapping, each parameter is measuring a somewhat different aspect of the same process and/or that each parameter is assessing a different subpopulation of what is globally referred to as 'lung destruction'. 
Our longitudinal study demonstrates that total and apoptotic EMP levels remain stable over a period of 1 year in subjects with no change to their smoking habits. Intervention with smoking cessation can normalise the levels of total and apoptotic EMPs in healthy smokers, but in contrast, cessation did not lead to significant changes in total and apoptotic EMP levels in COPD smokers in our study. This observation provides a biological correlate for epidemiological data showing that smoking cessation only has a moderate effect to slow the decline of lung function in COPD smokers, that is, we believe that these data show that EMP levels might serve as a useful biomarker to follow smoking-associated endothelial apoptosis. The longitudinal aspect of this study demonstrates persistent endothelial stress in subjects with COPD, despite smoking cessation, and may help to explain the irreversible lung destruction associated with most cases of COPD, as evidenced by lung function of COPD smokers following smoking cessation that does not return to normal, ${ }^{15}$ and may serve as a basis for additional studies of the mechanisms of the continuous pulmonary damage leading to this persistent EMP release.

\author{
Author affiliations \\ ${ }^{1}$ Department of Genetic Medicine, Weill Medical College of Cornell University, \\ New York, New York \\ ${ }^{2}$ Department of Pulmonary and Critical Care Medicine, Weill Medical College of \\ Cornell University, New York, New York \\ ${ }^{3}$ Department of Biological Statistics and Computational Biology, Cornell University, \\ Ithaca, New York, USA \\ ${ }^{4}$ Hoffmann-La Roche, Nutley, New Jersey, USA \\ ${ }^{5}$ Novartis \\ ${ }^{6}$ Eli Lilly and Company \\ ${ }^{7}$ PPD Labs \\ ${ }^{8}$ Achillion Pharmaceuticals, Inc \\ ${ }^{9}$ Boehringer Ingelheim Pharmaceuticals
}

Acknowledgements The authors thank M Elnashar, A Rogalski, E Blass, $S$ Mootoo and T Wilson for their help in processing blood samples; the Clinical Operations and Regulatory Affairs, Department of Genetic Medicine for help with these studies; and N Mohamed for help in preparing this manuscript. These studies were supported, in part, by P50HL084936, R01HL107882, U01HL121828, UL1 TR000457, UL1 RR02414 and Hoffmann-La Roche.

Contributors Conception and design: RGC. Acquisition and data interpretation: YS-B, MRS, AK, CG, AET, BG-H, RJK, CH, JGM, HB, SS, SGP, HH, GW, CSS, SV, JSF and RGC. Drafting of the manuscript: YS-B, MRS, AK, AET and RGC.

Funding F. Hoffman-La Roche, National Institutes of Health (P5OHL084936), (R01HL107882), (U01HL121828) (UL1 RR02414) and (UL1 TR000457).

Competing interests Hans Bitter, Sriram Sridhar, Sreekumar G. Pillai, Holly Hilton, Gerhard Wolff, Christopher S. Stevenson, Sudha Visvanathan and Jay S. Fine were all former employees of Hoffmann-La Roche.

Ethics approval Weill Cornell Institutional Review Board.

Provenance and peer review Not commissioned; externally peer reviewed.

\section{REFERENCES}

1 Global Initiative for Chronic Obstructive Lung Disease. 2011. http://www.goldcopd. com/ (accessed 30 Jul 2015).

2 Fletcher $\mathrm{C}$, Peto R. The natural history of chronic airflow obstruction. Br Med J 1977;1:1645-8.

3 McDonough JE, Yuan R, Suzuki M, et al. Small-airway obstruction and emphysema in chronic obstructive pulmonary disease. N Engl J Med 2011;365:1567-75.

4 Hogg JC, Senior RM. Chronic obstructive pulmonary disease-part 2: pathology and biochemistry of emphysema. Thorax 2002;57:830-4.

5 Barnes PJ. Mediators of chronic obstructive pulmonary disease. Pharmacol Rev 2004; 56:515-48.

6 Spurzem JR, Rennard SI. Pathogenesis of COPD. Semin Respir Crit Care Med 2005:26:142-53.

7 Cosio Piqueras MG, Cosio MG. Disease of the airways in chronic obstructive pulmonary disease. Eur Respir J Supp/ 2001;34:41s-9s.
8 Hogg JC. Pathophysiology of airflow limitation in chronic obstructive pulmonary disease. Lancet 2004;364:709-21.

9 Aoshiba K, Yokohori N, Nagai A. Alveolar wall apoptosis causes lung destruction and emphysematous changes. Am J Respir Cell Mol Biol 2003;28:555-62.

10 Plataki M, Tzortzaki E, Rytila P, et al. Apoptotic mechanisms in the pathogenesis of COPD. Int J Chron Obstruct Pulmon Dis 2006;1:161-71.

11 Rennard SI. Pathogenesis of chronic obstructive pulmonary disease. Pneumonol Alergol Pol 2011;79:132-8.

12 Gordon C, Gudi K, Krause A, et al. Circulating endothelial microparticles as a measure of early lung destruction in cigarette smokers. Am J Respir Crit Care Med 2011;184:224-32.

13 Danilov SM, Gavrilyuk VD, Franke FE, et al. Lung uptake of antibodies to endothelial antigens: key determinants of vascular immunotargeting. Am J Physiol Lung Cell Mol Physiol 2001;280:L1335-47.

14 Chandra D, Sciurba FC, Gladwin MT. Endothelial chronic destructive pulmonary disease (E-CDPD): is endothelial apoptosis a subphenotype or prequel to COPD? Am J Respir Crit Care Med 2011;184:153-5.

15 Scanlon PD, Connett JE, Waller LA, et al. Smoking cessation and lung function in mild-to-moderate chronic obstructive pulmonary disease. The Lung Health Study. Am J Respir Crit Care Med 2000;161:381-90.

16 Chironi GN, Boulanger CM, Simon A, et al. Endothelial microparticles in diseases. Cell Tissue Res 2009;335:143-51.

17 Paige M, Burdick MD, Kim S, et al. Pilot analysis of the plasma metabolite profiles associated with emphysematous Chronic Obstructive Pulmonary Disease phenotype. Biochem Biophys Res Commun 2011;413:588-93.

18 Strulovici-Barel Y, Omberg L, O'Mahony M, et al. Threshold of biologic responses of the small airway epithelium to low levels of tobacco smoke. Am J Respir Crit Care Med 2010;182:1524-32.

19 Koga H, Sugiyama S, Kugiyama K, et al. Elevated levels of VE-cadherin-positive endothelial microparticles in patients with type 2 diabetes mellitus and coronary artery disease. J Am Coll Cardiol 2005;45:1622-30.

20 Tushuizen ME, Nieuwland R, Rustemeijer C, et al. Elevated endothelial microparticles following consecutive meals are associated with vascular endothelial dysfunction in type 2 diabetes. Diabetes Care 2007;30:728-30.

21 Preston RA, Jy W, Jimenez JJ, et al. Effects of severe hypertension on endothelial and platelet microparticles. Hypertension 2003;41:211-17.

22 MacNee W. Oxidative stress and lung inflammation in airways disease. Eur J Pharmacol 2001;429:195-207.

23 Abboud RT, Vimalanathan S. Pathogenesis of COPD. Part I. The role of protease-antiprotease imbalance in emphysema. Int J Tuberc Lung Dis 2008; 12:361-7.

24 Horstman LL, Jy W, Jimenez JJ, et al. Endothelial microparticles as markers of endothelial dysfunction. Front Biosci 2004;9:1118-35.

25 Jimenez JJ, Jy W, Mauro LM, et al. Endothelial cells release phenotypically and quantitatively distinct microparticles in activation and apoptosis. Thromb Res 2003;109:175-80.

26 Werner N, Wassmann S, Ahlers P, et al. Circulating CD31+/annexin V+ apoptotic microparticles correlate with coronary endothelial function in patients with coronary artery disease. Arterioscler Thromb Vasc Biol 2006;26:112-16.

27 Mallat $Z$, Benamer $H$, Hugel $B$, et al. Elevated levels of shed membrane microparticles with procoagulant potential in the peripheral circulating blood of patients with acute coronary syndromes. Circulation 2000;101:841-3.

28 Simak J, Gelderman MP, Yu H, et al. Circulating endothelial microparticles in acute ischemic stroke: a link to severity, lesion volume and outcome. J Thromb Haemost 2006:4:1296-302.

29 Amabile N, Guérin AP, Leroyer $A$, et al. Circulating endothelial microparticles are associated with vascular dysfunction in patients with end-stage renal failure. J Am Soc Nephrol 2005;16:3381-8.

30 González-Quintero VH, Smarkusky LP, Jiménez JJ, et al. Elevated plasma endothelial microparticles: preeclampsia versus gestational hypertension. Am J Obstet Gynecol 2004;191:1418-24.

31 Amabile N, Heiss C, Real WM, et al. Circulating endothelial microparticle levels predict hemodynamic severity of pulmonary hypertension. Am J Respir Crit Care Med 2008;177:1268-75

32 Arteaga RB, Chirinos JA, Soriano AO, et al. Endothelial microparticles and platelet and leukocyte activation in patients with the metabolic syndrome. Am J Cardiol 2006;98:70-4.

33 Chirinos JA, Heresi GA, Velasquez $\mathrm{H}$, et al. Elevation of endothelial microparticles, platelets, and leukocyte activation in patients with venous thromboembolism. J Am Coll Cardiol 2005;45:1467-71.

34 Ayers L, Ferry B, Craig S, et al. Circulating cell-derived microparticles in patients with minimally symptomatic obstructive sleep apnoea. Eur Respir J 2009:33:574-80

35 Heiss C, Amabile N, Lee AC, et al. Brief secondhand smoke exposure depresses endothelial progenitor cells activity and endothelial function: sustained vascular injury and blunted nitric oxide production. J Am Coll Cardiol 2008;51:1760-71. 


\section{Smoking}

36 Luisetti M, Ma S, ladarola $\mathrm{P}$, et al. Desmosine as a biomarker of elastin degradation in COPD: current status and future directions. Eur Respir J 2008;32:1146-57.

37 Comandini A, Rogliani P, Nunziata A, et al. Biomarkers of lung damage associated with tobacco smoke in induced sputum. Respir Med 2009;103:1592-613.

38 Criner GJ, Cordova F, Sternberg AL, et al. The National Emphysema Treatment Trial (NETT): Part I: Lessons learned about emphysema. Am J Respir Crit Care Med 2011;184:763-70.
39 Kim V, Han MK, Vance GB, et al. The chronic bronchitic phenotype of COPD: an analysis of the COPDGene Study. Chest 2011;140:626-33.

40 Nishimura M, Makita $\mathrm{H}$, Nagai $\mathrm{K}$, et al. Annual change in pulmonary function and clinical phenotype in chronic obstructive pulmonary disease. Am J Respir Crit Care Med 2012;185:44-52.

41 Salzman SH. Which pulmonary function tests best differentiate between COPD phenotypes? Respir Care 2012;57:50-60.

42 Agustí A, Celli B. Avoiding confusion in COPD: from risk factors to phenotypes to measures of disease characterisation. Eur Respir J 2011;38:749-51. 